'Departamento de Bioquímica Clínica e Inmunología, Facultad de Farmacia, Universidad de Concepción, Concepción, Chile.

${ }^{2}$ Departamento de Estadística, Facultad de Ciencias Físicas y Matemáticas, Universidad de Concepción, Concepción, Chile.

${ }^{3}$ Departamento de Psicología, Facultad de Ciencias Sociales, Universidad de Concepción, Concepción, Chile.

${ }^{4}$ Departamento de Educación Física, Facultad de Educación, Universidad de Concepción, Concepción, Chile.

${ }^{5}$ Departamento de Pediatría, Facultad de Medicina, Universidad de Concepción, Concepción, Chile. aQuímico Farmacéutico, Magíster en Inmunología.

bBioquímico, Candidato a Magíster en Bioquímica Clínica e Inmunología. Ingeniero Matemático, Magíster en Estadística, Doctor en Ingeniería Eléctrica.

dPsicóloga, Magíster en Psicología de la Salud.

eProfesora de Educación Física, Magíster en Educación.

Financiado por: Proyecto Fondo Nacional de Desarrollo Regional (FNDRRegión del Bío-Bío, № 12FS024). Fundación de la Familia.

Recibido el 24 de agosto de 2014, aceptado el 2 de julio de 2015.

Correspondencia a: Paulina Bustos Araya Profesor Asociado, Departamento Bioquímica Clínica e Inmunología Facultad de Farmacia Universidad de Concepción, Chile.

Casilla 237, Universidad de Concepción Teléfonos: 56412203022 / 5641 2204439 pbustos@udec.cl

\section{Impacto del Programa de manejo de la obesidad Bright Bodies aplicado a niños y adolescentes chilenos}

\author{
PAULINA BUSTOS ${ }^{1, \mathrm{a}}$, JORGE ORIAS ${ }^{1, \mathrm{~b}}$, KATIA SÁEZ ${ }^{2, \mathrm{c}}$, \\ MARCELA MALDONADO ${ }^{3, \mathrm{~d}}$, LILIANA CUADRA ${ }^{4, \mathrm{e}}$, SYLVIA ASENJO $^{5}$
}

\section{Effects of the Bright Bodies Program in Chilean obese children}

\begin{abstract}
Background: Yale University's Bright Bodies Program consists on a lifestyle intervention, in areas such as nutrition and exercise, while focusing on behavior modification and family support. Aim: To evaluate the impact of the Program in Chilean children and adolescents with obesity who participated in the Program during 8 months. Material and Methods: The weight management Program was carried out during 8 months and consisted in weekly sessions directed by dietitians or psychologists and exercise sessions twice per week in charge of physical education teachers. The family component was based on sessions for parents or caregivers to achieve the same goals of children activities. Results: Twenty eight obese children aged $9.5 \pm$ 2 years completed the eight months of intervention. There was a significant $5 \%$ reduction of body mass index (BMI), a 15\% reduction of BMI $z$ score and a $2.9 \%$ reduction of waist circumference. Bioelectrical impedance showed a $9 \%$ reduction of percentage body fat and a $7 \%$ increase in lean body mass. Blood pressure, blood glucose, total and LDL cholesterol and triglycerides decreased significantly, without changes in HOMA-IR. The frequency of metabolic syndrome decreased from $36 \%$ at baseline to $18 \%$ at the end of the intervention. A $43 \%$ reduction in caloric intake and an improvement in physical condition was also observed. Conclusions: The Bright Bodies Program produced significant and positive changes on anthropometric and metabolic parameters in this group of children.
\end{abstract}

(Rev Med Chile 2015; 143: 1136-1143)

Key words: Intervention, studies; Obesity; Programmed instruction.
L a prevalencia de la obesidad ha aumentado progresivamente en todas las edades y constituye un problema de salud pública, tanto en los países desarrollados, como aquellos en vías de desarrollo.

En Chile, la Segunda Encuesta Nacional de Salud reveló una prevalencia de obesidad de 25,1\% y 39,3\% de sobrepeso en la población mayor de 15 años ${ }^{1}$. Un aumento sostenido de la prevalencia de obesidad también se ha observado en población pediátrica. Según datos del Ministerio de Salud
(MINSAL), entre los años 2005 y 2013, ésta aumentó de 7,1\% a 10,2\% en menores de 6 años. Estudios realizados en la Región del Bío-Bío demostraron una prevalencia de $9,2 \%$ en una población de 25.102 adolescentes, de edades entre 10 y 18 años $^{2}$. Otros datos publicados en la misma región revelan que la prevalencia de sobrepeso y obesidad fue $11,2 \%$ y $6,5 \%$, respectivamente, en una población de 32.514 escolares, de edades entre 5 y 18 años ${ }^{3}$.

Existe evidencia de la asociación de obesidad con enfermedades crónicas no transmisibles como 
la diabetes mellitus tipo 2, cuya presentación en adolescentes ha aumentado en forma paralela con la epidemia de obesidad ${ }^{4}$.

Distintos programas de manejo de la obesidad en población infantil se han desarrollado en diferentes centros. Revisiones sistemáticas de intervenciones educativas en niños de 6 a 12 años han demostrado que éstas son efectivas en el tratamiento de la obesidad, no así en su prevención ${ }^{5,6,7}$.

El Programa Bright Bodies de la Universidad de Yale consiste en una intervención sobre el estilo de vida, considerando aspectos nutricionales, de actividad física, con énfasis en la modificación conductual y el apoyo familiar. Este programa, destinado a niños y adolescentes, ha demostrado ser exitoso en controlar la obesidad y mejorar los parámetros cardiometabólicos a corto y largo plazo $^{8,9}$.

El objetivo de nuestro estudio fue evaluar el impacto de la aplicación del Programa Bright Bodies sobre parámetros antropométricos, metabólicos, hábitos alimentarios y de actividad física, en una población de niños y adolescentes chilenos portadores de obesidad.

\section{Métodos}

\section{Participantes}

Se evaluó el estado nutricional del total de la población de 612 escolares de enseñanza básica pertenecientes a las escuelas "Villa Centinela Sur" y "Escuela Nueva Los Lobos", de la ciudad de Talcahuano. Todas las familias de los escolares portadores de obesidad fueron invitadas a participar en el Programa Bright Bodies en el Centro de la Fundación de la Familia, ubicado en la población Nueva Los Lobos de Talcahuano. Esta fundación, sin fines de lucro, tiene como objetivo contribuir al fortalecimiento de las familias de sectores vulnerables, promoviendo estilos de vida saludable, mediante el desarrollo de diversos programas.

A esta convocatoria acudió un total de 109 escolares con obesidad, de los cuales 50 accedieron a participar, previa explicación detallada del Programa y firma del consentimiento informado por padres o tutores. Este estudio cuenta con la aprobación del Comité de Ética de la Facultad de Medicina de la Universidad de Concepción. El $70 \%$ de los escolares $(\mathrm{n}=35)$ participó los primeros 4 meses y $56 \%(n=28)$ completó el total de los 8 meses de asistencia al Programa.

\section{Programa Bright Bodies de la Universidad de Yale}

Este programa contempla actividades correspondientes a talleres de $40 \mathrm{~min}$ de nutrición o de modificación conductual una vez por semana, a cargo de nutricionista y psicólogo, y de sesiones de actividad física de 50 min, 2 veces por semana, dirigidas por un profesor de Educación Física.

El manejo nutricional excluye el concepto de "dieta" y se basa en la mejor elección de alimentos saludables, de preferencia con bajo contenido graso y en porciones moderadas ${ }^{10}$.

El Programa se extendió durante 8 meses, desde abril a noviembre del año 2013. Algunos de los temas tratados en las 12 sesiones nutricionales fueron: "determinando el tamaño de la porción", "qué hay en una etiqueta" y "¿qué hago entre comidas?”. Los componentes de la modificación conductual comprendieron: reconocimiento de sí mismo, establecimiento de metas, estrategias cognitivas, conductuales y manejo de contingencias. Algunos tópicos tratados en el total de 12 sesiones fueron: "aprendiendo a resolver problemas", "identificando amenazas" y "entendiendo recaídas".

El ejercicio físico en cada sesión incluía una etapa de calentamiento previo, seguida de un circuito dirigido de actividad física de intensidad moderada a alta, utilizando el peso corporal y sobrecarga con mancuernas, para finalizar con un trabajo libre en bicicletas elípticas, trotadoras, vídeo juego musical (Dance Revolution) y balones de Pilates, como vuelta a la calma.

El apoyo familiar se estableció a través de talleres para padres o tutores dirigidos por nutricionista o psicólogo, cuya temática estaba en concordancia con la abordada en los talleres para los niños. La intervención con los padres está destinada a facilitar el surgimiento de conductas de salud en la familia, potenciando el rol parental. Los primeros 2 meses, estos talleres se realizaron cada 15 días y posteriormente en forma mensual.

Los escolares participantes recibían vales en reconocimiento de sus logros, los que canjeaban por premios de acuerdo a un puntaje pre-establecido.

\section{Evaluación del Programa Bright Bodies}

El impacto del Programa se evaluó a través de la modificación en los parámetros antropométricos, metabólicos y modificación de los hábitos alimentarios y de actividad física. 
Esta evaluación se realizó previo a la aplicación del Programa y al término de los 8 meses. Los escolares con obesidad participantes fueron medidos en posición vertical con estadiómetro de precisión $0,1 \mathrm{~cm}$ y pesados en balanza TANITA BC418MA (Tanita Corporation, Tokio, Japón), mediante impedanciometría, con la cual simultáneamente se evaluó la cantidad de masa grasa y masa magra expresada en $\mathrm{kg}$ y la distribución porcentual de grasa corporal total y segmentaria de tronco, extremidades superiores e inferiores. Se calculó el índice de masa corporal (IMC) $(\mathrm{kg} /$ $\mathrm{m}^{2}$ ), su percentil de acuerdo a tablas del $\mathrm{CDC}^{11} \mathrm{y}$ el $z$-score. Se midió la circunferencia de cintura con cinta métrica inextensible con precisión de 0,1 $\mathrm{cm}$. La presión arterial se determinó con equipo digital (Welch Allyn Connex ${ }^{\circledR}$ ProBP ${ }^{\mathrm{TM}} 3400$, NY, USA), en posición sentado, en tres oportunidades y se obtuvo el promedio de las presiones sistólicas y diastólicas. Se consideró hipertensión arterial sistólica o diastólica cuando era superior al percentil 95 según el criterio del National High Blood Pressure Education Program (NHBPEP) Working Group on Children and Adolescents.

Para la determinación de los parámetros bioquímicos, se colectó $12 \mathrm{ml}$ de sangre por punción venosa, en ayunas. Las muestras de suero y plasma fueron alicuotadas y almacenadas a $-20^{\circ} \mathrm{C}$. La glicemia, el colesterol total (C-total), colesterol HDL (C-HDL), triglicéridos y alanino-aminotransferasa (ALT) se midieron utilizando métodos espectrofotométricos en equipo automatizado (Cobas C111, Roche, Rotkreuz, Suiza). El colesterol-LDL (C-LDL) se calculó por fórmula de Friedewald. Las insulinemias se cuantificaron por enzimainmunoensayo (DRG Diagnostics, NJ, USA). La resistencia a la insulina se evaluó por el HOMA-IR (homeostasis model assessment-insulin resistance: insulinemia $\mathrm{mU} / \mathrm{mL} \mathrm{x}$ glicemia $\mathrm{mg} / \mathrm{dL} / 405$ ).

El síndrome metabólico se definió de acuerdo al criterio de Cook, que considera la presencia de al menos tres de los siguientes parámetros: circunferencia de cintura $\geq$ percentil $90^{12}$, presión arterial $\geq$ percentil 90 , C- $\mathrm{HDL} \leq 40 \mathrm{mg} / \mathrm{dL}$, triglicéridos $\geq 110 \mathrm{mg} / \mathrm{dL}$ y/o glicemia basal $\geq 100 \mathrm{mg} / \mathrm{dL}^{13}$.

La modificación de los hábitos alimentarios se evaluó a través de la Encuesta de Tendencia de Consumo Cuantificada ${ }^{14}$.

La evaluación de la condición física de los niños y adolescentes fue realizada por el profesor de Educación Física al inicio y al final del Progra- ma, considerando la cantidad de repeticiones y el tiempo requerido para el ejercicio de cada grupo muscular: abdominales, porción superior e inferior, pectoral, tríceps braquial, cuádriceps, bíceps braquial y tríceps sural.

\section{Análisis estadístico}

Para realizar el análisis estadístico se utilizó el software estadístico SAS (SAS, versión 9.2-release Institute, Cary, NC, USA). Las variables numéricas fueron representadas por la media y desviación estándar y las categóricas por el porcentaje. Se utilizó el test t de Student para grupos pareados (test de Wilcoxon) para evaluar el efecto de la intervención. Se verificó la normalidad de las variables mediante la prueba de Shapiro-Wilk. En todos los casos, se utilizó un nivel de significancia de 0,05.

\section{Resultados}

La población total evaluada correspondió a 612 escolares, de edad promedio 9,2 $\pm 3,0$ años, de los cuales, $52,4 \%$ era de sexo masculino y $47,6 \%$ de sexo femenino. La evaluación del estado nutricional de estos escolares reveló que 3,8\% presentaba bajo peso, $51,3 \%$ normopeso, $21,1 \%$ sobrepeso y $23,9 \%$ obesidad, lo que implica que $45 \%$ era portador de malnutrición por exceso. De esta población, se evaluó a los 109 escolares con obesidad que respondieron a la convocatoria, cuya edad promedio era de $9,4 \pm 2,3$ años, el percentil del IMC promedio de $24,8 \pm 3,6 \mathrm{Kg} / \mathrm{m}^{2}$, IMC $z$-score de 2,09 $\pm 0,32$ y el porcentaje de grasa corporal total de $35,1 \pm 5,7 \%$. Esta población de escolares tenía un HOMA-IR promedio de $4,4 \pm$ 2,8 , con un valor de 3,6 $\pm 1,7$ en hombres y 5,3 \pm 2,4 en mujeres.

Entre los factores de riesgo cardiovascular, destaca la presencia de hipertensión arterial en $53 \%$ de los escolares, dislipidemia, producto de una disminución del colesterol-HDL, en 53\% y de un aumento de los triglicéridos en $44 \%$ de ellos. Considerando la sumatoria de estos factores de riesgo, de acuerdo al criterio de Cook, la prevalencia de síndrome metabólico fue de $44 \%$ en esta población de escolares portadores de obesidad.

La evaluación clínica, antropométrica y metabólica realizada a los 28 escolares, cuya edad promedio era 9,5 $\pm 1,9$ años, y que completaron los 8 meses de aplicación del Programa Bright Bodies, 
demostró una disminución significativa de todos los parámetros antropométricos, tales como, IMC, IMC $z$-score, percentil del IMC, circunferencia de cintura, porcentaje de grasa total, del tronco y de las 4 extremidades. Además, se observó disminución significativa tanto de la presión arterial sistólica como diastólica (Tabla 1). Los cambios antropométricos más relevantes se reflejaron en una reducción de 5\% del IMC, 15\% del IMC $z$-score, $3 \%$ de la circunferencia de cintura, $9 \%$ del porcentaje de grasa corporal total y aumento de $7 \%$ de la masa magra. La presión arterial sistólica se redujo en $11 \%$ y la diastólica en 7\% (Figura 1).

En relación al metabolismo de los hidratos de carbono, se observó disminución significativa de la glicemia; sin embargo, una mayor concentración

Tabla 1. Evaluación antropométrica basal y al término del Programa de manejo de la obesidad Bright Bodies de niños y adolescentes portadores de obesidad $(n=28)$

\begin{tabular}{|lccc|}
\hline Variable & $\begin{array}{c}\text { Basal } \\
\text { Media } \pm \text { DE }\end{array}$ & $\begin{array}{c}\text { Final } \\
\text { Media } \pm \text { DE }\end{array}$ & p \\
\hline Talla $(\mathrm{cm})$ & $138,9 \pm 11,6$ & $144,5 \pm 11,0$ & 0,0001 \\
\hline Peso $(\mathrm{kg})$ & $48,2 \pm 10,8$ & $49,6 \pm 10,6$ & 0,0195 \\
\hline IMC $\left(\mathrm{kg} / \mathrm{m}^{2}\right)$ & $24,7 \pm 2,6$ & $23,6 \pm 3,1$ & 0,0003 \\
\hline IMC z-score & $2,0 \pm 0,4$ & $1,7 \pm 0,5$ & $<0,0001$ \\
\hline IMC percentil & $97,3 \pm 2,2$ & $94,1 \pm 5,7$ & 0,0002 \\
\hline \% Grasa & $34,6 \pm 5,5$ & $31,6 \pm 5,9$ & $<0,0001$ \\
\hline Masa grasa (kg) & $16,9 \pm 5,4$ & $16,1 \pm 5,7$ & 0,0919 \\
\hline Masa magra (kg) & $31,3 \pm 6,8$ & $33,5 \pm 6,7$ & $<0,0001$ \\
\hline \% grasa pierna derecha & $41,3 \pm 5,9$ & $38,0 \pm 6,2$ & 0,0005 \\
\hline \% grasa pierna izquierda & $41,2 \pm 5,2$ & $38,4 \pm 6,1$ & $<0,0001$ \\
\hline \% grasa brazo derecha & $42,5 \pm 5,8$ & $40,6 \pm 5,9$ & 0,0062 \\
\hline \% grasa brazo izquierda & $45,0 \pm 6,4$ & $42,8 \pm 6,5$ & 0,0052 \\
\hline \% grasa tronco & $28,3 \pm 6,3$ & $25,9 \pm 5,3$ & 0,0026 \\
\hline Circunferencia de cintura (cm) & $84,6 \pm 7,9$ & $82,1 \pm 8,6$ & 0,0136 \\
\hline P. Sistólica (mm Hg) & $115,0 \pm 13,7$ & $102,6 \pm 9,9$ & $<0,0001$ \\
\hline P. Diastólica (mm Hg) & $66,6 \pm 7,2$ & $62,0 \pm 6,6$ & 0,0161 \\
\hline
\end{tabular}

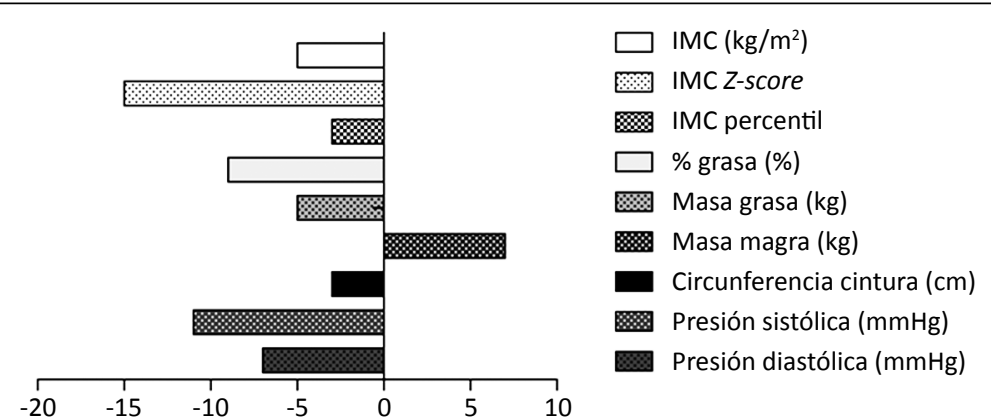

\% Diferencias entre Basal y Final

Figura 1. Diferencias de las variables antropométricas entre la evaluación basal y al término de la aplicación del Programa de manejo de la obesidad Bright Bodies en niños y adolescentes portadores de obesidad $(n=28)$. 
plasmática de insulina determinó ausencia de variación significativa del índice de resistencia a la insulina (HOMA-IR) (Tabla 2). Los parámetros lipídicos mostraron cambios favorables, reflejados en una disminución significativa del colesterol total, colesterol-LDL y triglicéridos, los que se redujeron en 23, 28 y $18 \%$, respectivamente. Sin embargo, los niveles de colesterol-HDL disminuyeron significativamente en $11,9 \%$ (Tabla 2).

La enzima hepática alanino-aminotransferasa (ALT), referida como indicador de inflamación hepática cuando se encuentra a altas concentraciones, se redujo en 13,6\%, sin alcanzar niveles de significancia (Tabla 2).

Inicialmente, del total de 28 niños y adolescentes obesos, $36 \%$ cumplía con el diagnóstico de síndrome metabólico, prevalencia que descendió a 18\% al término de la intervención (Figura 2).

En relación a los cambios de los hábitos alimentarios, la Encuesta de Tendencia de Consumo Cuantificado mostró modificaciones en el consumo de calorías totales, las que disminuyeron en $43 \%$, por reducción del aporte calórico de lípidos, carbohidratos y proteínas de $44 \%, 43 \%$ y $35 \%$, respectivamente.

La evaluación de la actividad física de los niños y adolescentes, realizada al inicio y al término del Programa, demostró que los mejores resultados globales se obtuvieron en los abdominales de la porción superior e inferior, donde $92 \%$ de los escolares optimizaron su rendimiento, seguido por $82 \%$ que mejoraron los músculos pectorales,

Tabla 2. Evaluación metabólica basal y al término del Programa de manejo de la obesidad Bright Bodies de niños y adolescentes portadores de obesidad $(n=28)$

\begin{tabular}{|lccc|}
\hline Variable & $\begin{array}{c}\text { Basal } \\
\text { Media } \pm \text { D.E. }\end{array}$ & $\begin{array}{c}\text { Final } \\
\text { Media } \pm \text { D.E. }\end{array}$ & p \\
\hline Glicemia $(\mathrm{mg} / \mathrm{dL})$ & $80,9 \pm 8,7$ & $70,5 \pm 5,2$ & $<0,0001$ \\
\hline Insulina $(\mathrm{mU} / \mathrm{mL})$ & $21,8 \pm 10,3$ & $30,6 \pm 14,8$ & 0,0013 \\
HOMA-IR & $4,4 \pm 2,2$ & $5,3 \pm 2,7$ & 0,0678 \\
\hline Colesterol total $(\mathrm{mg} / \mathrm{dL})$ & $152,7 \pm 30,8$ & $117,8 \pm 21,7$ & $<0,0001$ \\
\hline Colesterol-HDL $(\mathrm{mg} / \mathrm{dL})$ & $40,4 \pm 8,4$ & $35,6 \pm 10,5$ & 0,0061 \\
Colesterol-LDL $(\mathrm{mg} / \mathrm{dL})$ & $90,5 \pm 26,3$ & $65,5 \pm 18,3$ & $<0,0001$ \\
\hline Triglicéridos $(\mathrm{mg} / \mathrm{dL})$ & $102,3 \pm 52,2$ & $83,4 \pm 51,5$ & 0,0322 \\
ALT (U/L) & $21,3 \pm 13,5$ & $18,4 \pm 11,8$ & 0,2303 \\
\hline
\end{tabular}

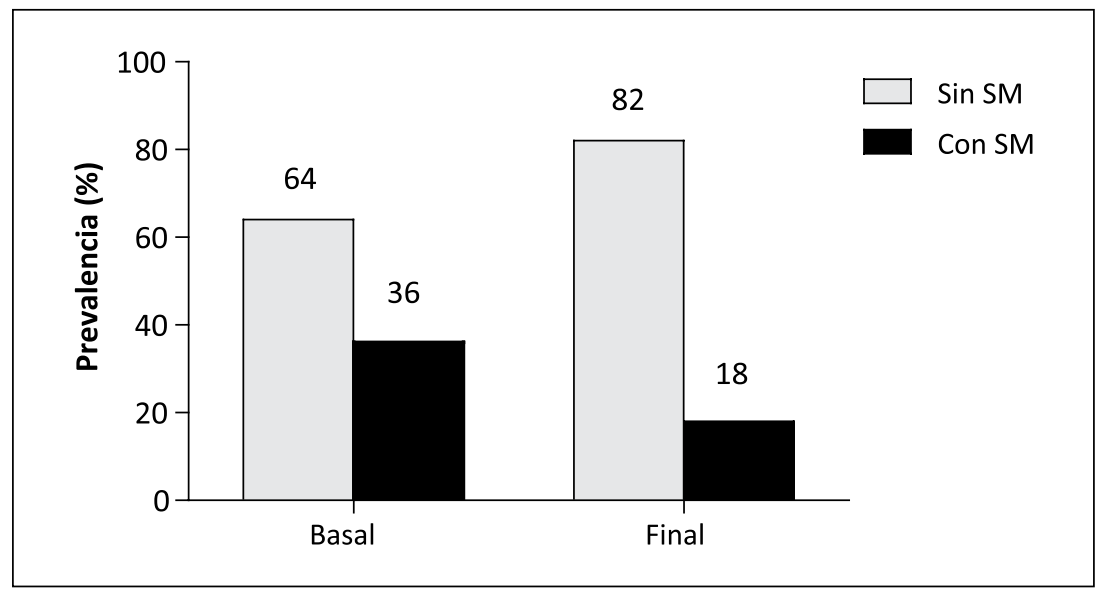

Figura 2. Prevalencia de síndrome metabólico de acuerdo al criterio de Cook en niños y adolescentes portadores de obesidad $(n=28)$. 
$80 \%$ los tríceps surales y $76 \%$ los bíceps braquiales. Los menores cambios se observaron en los tríceps braquiales y cuádriceps, donde 56 y 55\% de los escolares mejoró su condición, respectivamente.

\section{Discusión}

El Programa Bright Bodies de la Universidad de Yale, diseñado para diferentes etnias, ha sido aplicado preferentemente en distintos centros de Estados Unidos de Norteamérica (USA), en Escocia, Finlandia, y también por el equipo de profesionales responsables de este trabajo ${ }^{15}$.

Desde el año 2008, hemos desarrollado el Programa Bright Bodies en poblaciones de niños y adolescentes obesos de gran vulnerabilidad social con favorables resultados (XXIV Congreso SOCHED 2013). Este trabajo representa la labor realizada durante el año 2013 y corresponde a la primera publicación del impacto de este programa en niños y adolescentes obesos de nuestro país.

Los resultados demostraron que la aplicación del Programa Bright Bodies produjo resultados positivos, tanto en los parámetros antropométricos como metabólicos en niños y adolescentes obesos, al cabo de 8 meses de seguimiento. Cabe destacar que la reducción de 5\% del IMC y de 15\% del IMC $z$-score, representa un cambio significativo e importante del estado nutricional y de la composición corporal, dada por la disminución del porcentaje de grasa corporal total, lo que significa una diferencia de -3. Datos publicados del Programa Bright Bodies realizado en la Clínica de Obesidad Pediátrica de Yale, New Haven, USA, aplicado en una población de niños obesos de 8 a 16 años de edad, de diferentes etnias y que completaron 12 meses de seguimiento, la diferencia del porcentaje de grasa corporal total fue de -4 y para el IMC de $-1,7$, cifras comparables a la diferencia de -3 y de $-1,1$, respectivamente, obtenidas en el grupo de niños y adolescentes chilenos considerando el desarrollo del Programa durante sólo 8 meses ${ }^{16}$.

En relación al impacto del Programa Bright Bodies sobre los cambios metabólicos, se encontró disminución significativa de la glicemia basal sin cambios significativos del HOMA-IR. Estos resultados contrastan con lo referido al aplicar este Programa en población de niños y adolescentes en USA, que demostró ser efectivo en aumentar la sensibilidad a la insulina, reducir las glicemias post-carga de glucosa y el riesgo de diabetes tipo 2 en adolescentes obesos ${ }^{9,17}$. Por el contrario, encontramos cambios favorables y significativos en los valores de colesterol total, colesterol-LDL y triglicéridos de los niños y adolescentes obesos participantes del Programa. Sin embargo, los niveles de colesterol-HDL también disminuyeron significativamente, aunque para una interpretación completa de la modificación de este parámetro lipídico sería importante conocer además la composición de las apolipoproteínas, principalmente de la apo AI. Al comparar el impacto sobre el metabolismo lipídico del Programa Bright Bodies en USA, se observó igualmente disminuciones significativas de los triglicéridos y del colesterol total, sin variaciones significativas en los niveles de los otros lípidos a los 6 meses de tratamiento ${ }^{9,16}$.

La ALT, utilizada como marcador de enfermedad hepática no alcohólica cuando se encuentra a altas concentraciones, se redujo sin alcanzar niveles de significancia, al igual que lo demostrado por Savoye et $\mathrm{al}^{9}$. Recientemente, se ha informado que niveles de ALT mayores de 19 UI/L y de triglicéridos mayores de $101 \mathrm{mg} / \mathrm{dL}$ se encuentran estrechamente relacionados con enfermedad hepática no alcohólica ${ }^{18}$. Estos parámetros en los niños y adolescentes eran superiores a dichos valores antes de participar en el Programa Bright Bodies, y disminuyeron siendo menores a las concentraciones antes mencionadas al término de los 8 meses. Estos cambios metabólicos alcanzados son importantes, pues se ha demostrado asociaciones significativas entre enfermedad hepática no alcohólica con marcadores de obesidad visceral y resistencia a la insulina en ambos sexos ${ }^{19}$.

El síndrome metabólico es altamente prevalente en niños y adolescentes obesos y representa un factor de riesgo de diabetes mellitus tipo $2 \mathrm{y}$ de enfermedad cardiovascular. Inicialmente, $36 \%$ de los niños y adolescentes obesos presentaba síndrome metabólico, prevalencia que descendió a $18 \%$ al término de la intervención. Esta respuesta concuerda con lo demostrado en otros estudios, donde la reducción de la obesidad contribuye a un aumento de la sensibilidad a la insulina y, en consecuencia, a revertir el síndrome metabólico. Resultados obtenidos en un estudio longitudinal de alrededor de 2 a 3 años, demostraron que 20 a $50 \%$ de adolescentes obesos que presentaban síndrome metabólico, cambiaron dicha condición debido a que perdieron o mantuvieron el peso ${ }^{20}$. 
Los beneficios del Programa Bright Bodies se observaron también en los cambios de los hábitos alimentarios, con una disminución de $43 \%$ en el consumo de calorías totales y un mejoramiento de la actividad física al evaluar 7 grupos musculares en los niños y adolescentes obesos. El cambio en la ingesta nutricional no se puede comparar con los resultados publicados por los autores del Programa Bright Bodies, dado que ellos no registran el consumo de calorías y macronutrientes, pues deliberadamente omiten el registro de la ingesta alimentaria con el propósito de evitar el concepto de "dieta". Además, una situación similar ocurre en relación a los cambios en la actividad física, lo que no permite comparar estos resultados ${ }^{8,16}$.

El 56\% de los niños completó los 8 meses del Programa Bright Bodies, cifra superior a lo reportado en otras publicaciones que indican que $53 \%$ de los niños que consulta por obesidad abandona dentro de los primeros 6 meses $^{21}$. En otra experiencia nacional, la adherencia alcanzó a $18 \%$ a un año plazo, valor comparable a la de otros estudios convencionales ${ }^{21}$. Considerando estas cifras, estimamos satisfactoria la adherencia a este Programa desarrollado en niños provenientes de una población de vulnerabilidad social y que participan de actividades ofrecidas por la Fundación de la Familia.

El Programa Bright Bodies aplicado en niños obesos de distintas etnias ha demostrado que los efectos benéficos de los parámetros antropométricos y metabólicos, incluidos el aumento de la sensibilidad a la insulina y la mejoría de la tolerancia a la glucosa, se mantienen después de 12 meses de completar la fase de intervención activa ${ }^{8}$. Además, se señala que los efectos positivos sobre el peso, la composición corporal, lípidos plasmáticos, insulina y sensibilidad a la insulina se mantuvieron aún por 24 meses, lo cual indica que la educación, la modificación conductual y el entrenamiento físico tuvieron un efecto de larga duración. Estos resultados fueron citados en la última revisión Cochrane, indicando al Programa Bright Bodies como una de las mejores intervenciones con modificaciones del estilo de vida en niños y adolescentes ${ }^{6}$.

La aplicación del Programa Bright Bodies en una población de 28 niños y adolescentes chilenos portadores de obesidad produjo cambios favorables y significativos sobre los parámetros antropométricos, metabólicos, hábitos alimentarios y de actividad física. Sin embargo, algunas limitaciones del estudio se refieren a que no permite proyectar los efectos beneficiosos a largo plazo, lo cual sí ha sido demostrado en estudios de seguimiento por los autores del Programa Bright Bodies, que comprobaron que éstos se mantienen al cabo de 2 años ${ }^{8}$. Además, otro aspecto a considerar es la evaluación del costo por niño intervenido para la amplia aplicación de este Programa.

Agradecimientos: A la Fundación de la Familia, Sra. Catalina Recordon Martin, Directora, años 2010 a 2014. A la Señora María Cristina Escobar Contreras, Nutricionista participante en el Programa.

\section{Referencias}

1. Ministerio de Salud. Encuesta Nacional de Salud 20092010, http://epi.minsal.cl/epi/html/invest/ENS/ENS. htm. (Consultado el 27 de abril de 2014).

2. Bustos P, Sáez K, Gleisner A, Ulloa N, Calvo C, Asenjo S. Metabolic syndrome in obese adolescents. Pediatr Diabetes 2010; 11 (1): 55-60.

3. Ulloa N, Sapunar J, Bustos P, Sáez K, Asenjo S, Taibo $\mathrm{M}$, et al. Epidemiología del sobrepeso y obesidad infanto-juvenil en las comunas de Concepción, Coronel y Hualpén, VIII Región de Chile. Rev Med Chile 2010; 138 (11): 1365-72.

4. Caprio S. The development of type 2 diabetes in the obese adolescents: a growing challenge. Clin Biochem 2014; 47 (9): 721-95.

5. Sbruzzi G, Eibel B, Barbiero S, Petkowicz R, Ribeiro R, Cesa $\mathrm{C}$, et al. Educational interventions in childhood obesity: a systematic review with meta-analysis of randomized clinical trials. Prev Med 2013; 56 (5): 254-64.

6. Oude Luttikhuis H, Baur L, Jansen H, Shrewsbury VA, O'Malley C, Stolk RP, et al. Interventions for treating obesity in children. Cochrane Database Syst Rev 2009; 21 (1): CD001872.

7. Janicke D, Steele R, Gayes L, Lim C, Clifford L, Schneider $\mathrm{E}$, et al. Systematic review and meta-analysis of comprehensive behavioral family lifestyle interventions addressing pediatric obesity. J Pediatr Psychol 2014; 39 (8): 809-25.

8. Savoye M, Nowicka P, Shaw M, Yu S, Dziura J, Chavent $\mathrm{G}$, et al. Long-term results of an obesity program in an ethnically diverse pediatric population. Pediatrics 2011; 127 (3): 402-10.

9. Savoye M, Caprio S, Dziura J, Camp A, Germain G, Summers C, et al. Reversal of early abnormalities in glucose metabolism in obese youth: results of an inten- 
sive lifestyle randomized controlled trial. Diabetes Care 2014; 37 (2): 317-24.

10. Bright Bodies Weight Management Program for Children. Available from: http://www.brightbodies.org/

11. Centers for Disease Control and Prevention: CDC Growth Charts: U.S. <http://www.cdc.gov/growthcharts/ data_tables.htm > (2009) (Accessed 24 april 2014).

12. Fernández JR, Redden DT, Pietrobelli A, Allison DB. Waist circumference percentiles in nationally representative samples of african-american, european-american and mexican-american children and adolescents. J Pediatr 2004; 145: 439-44.

13. Cook S, Weitzman M, Auinger P, Nguyen M, Dietz W. Prevalence of a metabolic syndrome phenotype in adolescents: findings from the third National Health and Nutrition Examination Survey, 1988-1994. Arch Pediatr Adolesc Med 2003; 157 (8): 821-7.

14. Urteaga C, Pinheiro A. Investigación alimentaria: consideraciones prácticas para mejorar la confiabilidad de los datos. Rev Chil Nutr 2003; 30 (3): 235-42.

15. Smart Moves ${ }^{\mathrm{TM}}$. Available from: http://www.smartmovesforkids.com/smart-moves-bright-bodies.html.

16. Savoye M, Shaw M, Dziura J, Tamborlane WV, Rose $\mathrm{P}$, Guandalini C, et al. Effects of a weight management program on body composition and metabolic parameters in overweight children: a randomized controlled trial. JAMA 2007; 297 (24): 2697-704.

17. Shaw M, Savoye M, Cali A, Dziura J, Tamborlane WV, Caprio S. Effect of a successful intensive lifestyle program on insulin sensitivity and glucose tolerance in obese youth. Diabetes Care 2009; 32 (1): 45-7.

18. Tomizawa M, Kawanabe Y, Shinozaki F, Sato S, Motoyoshi Y, Sugiyama T, et al. Elevated levels of alanine transaminase and triglycerides within normal limits are associated with fatty liver. Exp Ther Med 2014; 8 (3): 759-62.

19. Denzer C, Thiere D, Muche R, Koenig W, Mayer H, Kratzer W, et al. Gender-specific prevalences of fatty liver in obese children and adolescents: roles of body fat distribution, sex steroids, and insulin resistance. J Clin Endocrinol Metab 2009; 94 (10): 3872-81.

20. Weiss R, Shaw M, Savoye M, Caprio S. Obesity dynamics and cardiovascular risk factor stability in obese adolescents. Pediatr Diabetes 2009; 10 (6): 360-7.

21. Barja S, Núñez E, Velandia S, Urrejola P, Hodgson MI. Adherencia y efectividad a mediano plazo del tratamiento de la obesidad infantil. Rev Chil Pediatr 2005; 76 (2): 151-8. 